\title{
Effects of Acute Psychosocial Stress on Facial Emotion Recognition
}

\author{
Efrat Barel, Ami Cohen \\ Emek Yezreel College, Jezreel Valley, Israel \\ Email: efratb@yvc.ac.il
}

How to cite this paper: Barel, E., \& Cohen, A. (2018). Effects of Acute Psychosocial Stress on Facial Emotion Recognition. Psychology, 9, 403-412.

https://doi.org/10.4236/psych.2018.93025

Received: February 18, 2018

Accepted: March 27, 2018

Published: March 30, 2018

Copyright (C) 2018 by authors and Scientific Research Publishing Inc. This work is licensed under the Creative Commons Attribution International License (CC BY 4.0).

http://creativecommons.org/licenses/by/4.0/

\begin{abstract}
Accurate recognition of the emotions of other people is essential for successful human interactions. Previous studies showed inconsistent results of the impact of acute psychosocial stress on emotion recognition. Thus, the present study aimed at investigating the influence of psychosocial stress on the recognition of facial emotional expressions of different valance. Eighty-eight participants were randomly assigned to one of two experimental groups: stress (exposure to the Trier Social Stress Test) and control. Participants of both groups completed a facial emotion recognition test before and after the manipulation. The results demonstrated an enhancement in emotion recognition in the stress group but not in controls. Further analyses revealed that while stress enhanced the accurate recognition of anger, happiness, surprise, and neutral/calmness, it impaired the recognition of fear and had no effect on the recognition of sadness and disgust. The findings are discussed in light of evolutionary assumptions regarding differential processing mechanisms of emotion expressions.
\end{abstract}

\section{Keywords}

Stress, Facial Emotion Recognition, TSST

\section{Introduction}

Success in social interaction greatly depends on individuals' social cognition. Social cognition refers to the mental operations underlying social relationships and includes the capacity to understand and attribute ourselves as well as others with feelings, thoughts, and beliefs (Smeets, Dziobek, \& Wolf, 2009). Two of the main domains of social cognition are empathy and facial emotion recognition (Cusi, Nazarov, Holshausen, Macqueen, \& McKinnon, 2012). Empathy is a complex construct involving the capacity to understand the perspective and intentions of 
others (Baron-Cohen, 2003), whereas facial emotion recognition is a more basic ability to accurately perceive emotions. It is assumed that facial expression evolved in cooperative societies and has numerous adaptive consequences (Sacco \& Hugenberg, 2009), including the ability to accurately identify threat signals and therefore select avoidance strategy (Brown \& Moore, 2002), and the ability to accurately perceive displays of fear in order to better communicate danger (Reed \& DeSciolo, 2017).

Importantly, the processes underlying social cognition may be altered under conditions of stress as do many human attentional, perception, and response mechanisms (Staal, 2004). Indeed, stress-induced alterations in learning and memory are well-documented in both humans and laboratory animals (LaBar \& Cabeza, 2006; Lupien, Maheu, Tu, Fiocco, \& Schramek, 2007; Roozendaal, Okuda, de Quervain, \& McGaugh, 2006; Schoof, Preuß, \& Wolf, 2008; Shields, Sazma, \& Yonelinas, 2016; Wolf, 2006). Some of these studies found enhancement and some found impairment in performance, an inconsistency that may be partly explained by variables such as the type and magnitude of the stressor used in various studies. However, the impact of stress on the different aspects of social cognitions has been less extensively studied.

Studies focusing on facial emotion recognition following exposure to stress show inconsistent results, with some demonstrating higher accuracy on emotion recognition after stress induction compared to baseline, while others do not provide support to this finding. For example, in a study using the cold pressor test (CPT) - a psychophysiological stressor in which participants are instructed to place their hand in ice-cold water, men who scored low on the hostility scale and who were stressed through the CPT perform slightly worse in facial emotion recognition task than non-stressed participants (Herridge, Harrison, Mollet, \& Shenal, 2004).

Another line of studies focused on the use of psychosocial stressors. Psychosocial stress is defined as a moderate stress, and is commonly used in human studies due to its relevance to what humans encounter on a daily basis (Corbett, Weinberg, \& Duarte, 2017). In an Internet-based experiment, participants were randomly assigned to the experimental groups (stress, support) or the control group. The stress manipulation was induced through the feedback and the formulation of instruction each group received. Stress-impaired facial emotion recognition was found in the stress group compared with the controls (Hänggi, 2004). Particularly common is the use of the Trier Social Stress Test (TSST; Kirschbaum Pirke, \& Hellhammer, 1993), the most validated tool for inducing psychosocial stress (Skoluda et al., 2015). The TSST consists of a free speech task and a mental arithmetic task in front of an audience. Studies examining the impact of the TSST on emotion recognition provide inconsistent results. Specifically, Deckers et al. (2015) and colleagues found TSST-induced enhancement in emotion recognition on various emotional facial expressions (anger, disgust, fear, happiness, sadness, and surprise) in healthy participants as well as in pa- 
tients with borderline personality disorder; while Li, Weerda, Milde, Wolf, \& Theil (2014), examining the identification of neutral and fearful expressions, found no evidence for such enhancement. Furthermore, Wolf et al. (2015) found that while exposure to the TSST enhanced emotional empathy (which describes the participant's emotional response to another person's emotional state), it had no effect on emotion recognition.

The adaptive mechanisms of human processing of emotional stimuli under stress circumstances are crucial for our survival. Given the inconsistencies in previous studies, the current study aimed at investigating the impact of acute psychosocial stress exposure, using the TSST, on facial emotion recognition. Furthermore, the present study aimed at differentiating between different facial expressions, as it has been proposed that there is an evolutionary advantage in attending to negative emotional stimuli (negativity bias) in order to detect and survive threats (Hamann, 2001). Previous studies provided support for this assertion showing that threatening faces, such as angry faces, are attended more than positive or neutral faces (Bannerman, Milders, \& Sahraie, 2010; Huang, Chang, \& Chen, 2011). However, studies investigating the negativity bias following stress provided mixed results. For example, Oei (2012) and colleagues found enhanced negativity bias in participants following the TSST, whereas Wolf et al. (2015) did not demonstrate any valence-specific effects, with participants demonstrating enhancement in affective empathy for both positive and negative emotions. van Marle, Hermans, Qin, \& Fernández (2009) attributed amygdala shifts from selective response to angry and fearful faces in the control group to indiscriminative response to happy faces as well as angry and fearful faces in the stress-exposed group. The authors proposed that the automatic processing of the amygdala concerning potentially threatening signals from the environment often results in false positive errors, especially under stress conditions. In particular, happy faces are commonly portrayed as "flashing" exposure of white teeth, and thus may be processed as potentially threatening. In sum, the hypothesis of the current study was that exposure to the TSST will enhance the detection of potentially threatening emotions (angry, surprised, happy) while not affecting the detection of non-threatening stimuli (neutral, calm). As fearful facial expressions are negative but do not indicate a direct threat posed by the person expressing it (Marsh, Ambady, \& Kleck, 2005), we predicted that the TSST will not affect the detection of this emotion.

\section{Methods}

\subsection{Participants}

Eighty-eight men and women participated in the present study. The mean age was 23.77 ( $\mathrm{SD}=2.90)$. Participants were recruited from among college students by advertisements. After signing an informed consent form, the volunteers completed a questionnaire regarding their health, habits, and demographic details to verify that they met the inclusion criteria: no serious medical, gynecological, or 
hormonal problems; non-smokers. The Institutional Ethics Review Board approved the complete study protocol.

\subsection{Experimental Procedure}

Experimental sessions took place in the laboratory of the Psychology Department between $8 \mathrm{AM}$ and $10 \mathrm{AM}$. Participants were randomly assigned to one of two experimental groups: stress $(N=45)$ and control $(N=43)$. The stress session comprised the following three consecutive stages: 1$)$ completion of the facial emotion recognition task (5 minutes); 2) the Trier Social Stress Test procedure (20 minutes); and 3) completion of the same task as stage A (20 minutes). The non-stress control group completed a non-stressful control task of equal duration (reading the entry "England" in Wikipedia silently, reading the entry "transport in Israel" out loud, and counting out loud, but alone in a room).

\subsection{Trier Social Stress Test}

Psychological stress was induced by employing the TSST procedure (Kirschbaum et al., 1993). This procedure consists of a stress task that includes 5 minutes of free speech (a simulated job interview for the participant's "dream job") and 5 minutes of a mental arithmetic task, both conducted in front of a committee composed of a man and a woman sitting at a distance of $1.5 \mathrm{~m}$ and a video camera. At the beginning of the procedure, the participants were instructed by the committee regarding the task at hand, were notified that the performance will be recorded for subsequent behavioral analysis, and then taken to a second room in which they had 10 minutes to formulate the speech alone. Next, the participants entered the committee room in which they carried out the free speech task and the arithmetic task. In total, the procedure, including the preparation phase, took approximately 20 minutes.

\subsection{Facial Emotion Recognition Task}

The emotion recognition task measures six basic emotional expressions: anger, fear, disgust, surprise, sadness, and happiness, together with two expressions: calmness and neutral (calculated as one score: Markovits, Trémolière, \& Blanchette, 2018). The faces were taken from the NIMSTIM (Tottenham et al., 2009). The task included 20 trials presented in random order (conducted through three different versions). On each trial participants were requested to select one of four possible answers. Correct responses were summed.

\subsection{Statistical Analyses}

Emotion recognition scores before and after the TSST were not normally distributed and were thus subject to $\log 10$ transformation that normalized their distribution.

Mixed ANOVA was used to test the hypothesis regarding stress impact on emotion recognition scores with time (pre- and post-TSST) as a within subject 
independent variable, and group (stress, control) as a between subject independent variable, and with "version" as covariate.

In order to test the hypothesis regarding the differential influence of stress on each emotion, recognition scores on each emotion were analyzed via series of repeated measures analyses of variance (time $\times$ group).

In order to examine former suggestions regarding a female advantage in emotion recognition (e.g., Kirkland, Peterson, Baker, Miller, \& Pulos, 2013), a series of three-way repeated-measures analyses of variance with sex (male, female) $\times$ group (stress, control) $\times$ time (before and after TSST) on emotion recognition (general and differential) were conducted.

\section{Results}

To examine whether stress influenced general emotion recognition performance a two-way repeated-measures analysis of variance with group (stress vs. control) $\times$ time (before and after TSST or the control manipulation) and test version as covariate was conducted. The interaction group $\times$ time for general emotion recognition performance was significant $\left[F(1,85)=28.13, p<0.001 ; \eta_{p}^{2}=0.25\right]$. Decomposing the interaction revealed a significant effect of time on emotion recognition performance in the stress group $[t(44)=6.44, p<0.001$, Cohen's $d=$ 1.00]. Performance of the stress group was enhanced after stress induction $(\mathrm{M}($ pre $)=1.23, \mathrm{SD}=0.03 ; \mathrm{M}($ post $)=1.26, \mathrm{SD}=0.03)$, whereas no significant difference $[t(42)=1.77, p>0.05$, Cohen's $d=0.25]$ was found in the control group $(\mathrm{M}($ pre $)=1.25, \mathrm{SD}=0.03 ; \mathrm{M}($ post $)=1.24, \mathrm{SD}=0.04)$.

To examine whether stress influenced each specific emotion recognition performance a series of two-way repeated-measures analyses of variance with group (stress vs. control) $\times$ time (before and after TSST or the control manipulation) and test version as covariate were conducted. The interaction group $\times$ time for fear recognition was significant $\left[F(1,72)=5.16, p<0.05 ; \eta_{p}^{2}=0.07\right]$. Decomposing the interaction revealed a significant effect of time on fear recognition in the stress group, but not in the control group. Performance of the stress group deteriorated after stress induction (see Table 1). The interaction effects for anger, happiness, surprise, and neutral/calmness were also significant $[F(1,84)=$ $14.02, p<0.001 ; \eta_{p}^{2}=0.14 ; F(1,85)=21.49, p<0.001 ; \eta_{p}^{2}=0.20 ; F(1,82)=$ 13.81, $p<0.001 ; \eta_{p}^{2}=0.14 ; F(1,85)=6.96, p<0.05 ; \eta_{p}^{2}=0.08$, respectively]. The pattern of change was reversed: performance was enhanced after stress induction in the stress group, whereas no significant effect was found for the control group. The interaction effects for sad and disgust were not significant $[F(1$, $83)=3.37, p>0.05 ; \eta_{p}^{2}=0.04 ; F(1,84)=2.15, p>0.05 ; \eta_{p}^{2}=0.03$, respectively].

\section{Sex Differences}

A series of three-way repeated-measures analyses of variance with sex (male, female) $\times$ group (stress, control) $\times$ time (before and after TSST) on emotion 
Table 1. Means (SD), SD, paired samples t-test, and effect sizes (Cohen's d).

\begin{tabular}{|c|c|c|c|c|c|c|c|c|}
\hline & \multicolumn{2}{|c|}{ Stress } & \multirow{2}{*}{$t$} & \multirow{2}{*}{$\mathrm{d}$} & \multicolumn{2}{|c|}{ Control } & \multirow{2}{*}{$-t$} & \multirow{2}{*}{ d } \\
\hline & Pre-TSST & Post-TSST & & & Pre-manipulation & Post-manipulation & & \\
\hline Fear & $0.45(0.09)$ & $0.28(0.18)$ & $5.02^{* * *}$ & 0.76 & $0.24(0.16)$ & $0.18(0.18)$ & 1.89 & 0.33 \\
\hline Anger & $0.35(0.14)$ & $0.44(0.09)$ & $3.68^{\star *}$ & 0.05 & $0.45(0.06)$ & $0.43(0.11)$ & 1.1 & 0.20 \\
\hline Happiness & $0.36(0.11)$ & $0.46(0.05)$ & $5.1^{* * *}$ & 0.77 & $0.46(0.06)$ & $0.45(0.06)$ & 0.37 & 0.00 \\
\hline Sadness & $0.23(0.13)$ & $0.27(0.10)$ & 1.63 & 0.24 & $0.29(0.05)$ & $0.28(0.08)$ & 1.43 & 0.02 \\
\hline Disgust & $0.41(0.09)$ & $0.46(0.08)$ & $2.88^{\star *}$ & 0.50 & $0.45(0.06)$ & $0.46(0.08)$ & 0.33 & 0.09 \\
\hline Surprise & $0.21(0.14)$ & $0.30(0.00)$ & $4.29^{* * *}$ & 0.64 & $0.30(0.00)$ & $0.30(0.00)$ & 0.00 & 0.00 \\
\hline $\begin{array}{l}\text { Neutral/ } \\
\text { Calmness }\end{array}$ & $0.23(0.09)$ & $0.28(0.05)$ & $2.69^{\star}$ & 0.11 & $0.28(0.05)$ & $0.27(0.06)$ & 1.08 & 0.17 \\
\hline
\end{tabular}

recognition (general and differential) were conducted. No significant main effect for sex was found, nor sex $\times$ group $\times$ time interaction for general as well as for each emotion recognition $(p>0.05)$.

\section{Discussion}

The present study aimed at investigating the impact of stress exposure on emotion recognition enhancement. Specifically, the present study investigated whether exposure to acute psychosocial stress influences general emotion recognition, and further investigated whether this enhancement pattern is unified across emotions, or differentiated to facial expressions associated with potential threat signals to the individual.

The present findings showed that exposure to acute psychosocial stress generally enhances facial emotion recognition. This is in line with previous findings demonstrating enhancement in emotion recognition on various emotional expressions (anger, disgust, fear, happiness, sadness, and surprise) in healthy participants as well as in patients with borderline personality disorder following the TSST (Deckers et al., 2015). However, some studies did not show enhancement in emotion recognition following exposure to acute stress (for example, Li et al., 2014). Future studies should pursue investigating the specific conditions under which the enhancement in emotion recognition takes place. Given the diversity in stressors used (e.g., TSST vs. CPT) and emotion recognition tests (e.g., Reading in the Eyes Test: Baron-Cohen, Wheelwright, Hill, Raste, \& Plumb, 2001, vs. facial emotion recognition tasks), future studies should control these variables in order to uncover the influence pattern of acute stress on emotion recognition. It is important to note that while the present study investigated the potential role of acute stress on emotion recognition enhancement, studies focusing on the influence of chronic stress found an inhibitory effect rather than enhancement. For example, studies on individuals exposed to early traumatic experiences showed deficits in emotion recognition including deviant brain response (e.g., Curtis \& Cicchetti, 2013; Lieslehto et al., 2017). Overall, the findings of the 
present study suggest that acute stress results in greater attendance to emotional cues, which is in line with the evolutionary framework suggesting that the ability to detect and assess potential threats under adverse conditions is essential to our survival (McEwen, 2007).

Participants in the stress group showed enhancement in emotion recognition of anger, happiness, surprise, and neutral/calmness emotions, whereas performance worsened for fear. Stress did not affect performance for sadness and disgust. The findings concerning the enhanced detection of anger and surprise are partly in line with previous findings suggesting elevations in accuracy of threat-related signals following stress. For example, Bechtoldt \& Schneider (2016) found higher accuracy in recognition of fear and anger (high-arousal negative emotions) compared to sadness and disgust (low-arousal negative emotions). In the present study, however, deterioration in performance was found for fear. Perhaps a fine-tuning distinction in threat-related stimuli is needed. Detection of anger is crucial for survival. Therefore the enhancement found in the present study as well as in former studies is in line with the evolutionary framework suggestion as an adaptive mechanism of detection of threat-related signals. The findings regarding fear are more difficult to explain. Fearful signals suggest the existence of an indirect potential threat in the environment but do not contain direct threat signals to the individual. This finding is in line with previous studies in animals and humans, showing differential processing mechanisms for fear and anger expressions. Although fear and anger facial expressions are both perceived as containing threat signals, results in animals and humans suggest that they elicit divergent responses. For example, subordination or fear displays in social species appear nonthreatening and keep them from dominant members' aggression (Blair, 1995; Preuschoft, 1999). Marsh et al. (2005) and colleagues found that anger and fear expressions affected opposite motoric responses among students. While anger expressions resulted in responses associated with avoidance-related behaviors, fear expressions resulted in responses associated with approach-related behaviors. The enhanced performance on happy faces detection in the stressed group was also unexpected. However, van Marle et al. (2009) suggested that happy faces are portrayed as "flashing" exposure of white teeth, and may be processed as potentially threatening to the stressed individual. They have demonstrated amygdala shifts from selective response to angry and fearful faces in the control group, to indiscriminative response of happy faces as well in the experimental group exposed to stress.

\section{Conclusion}

In sum, an emotion-dependent enhancement or deterioration in performance was found in the present study, suggesting that the impact of stress on emotion recognition is not unified across emotions. Since emotion recognition is a core component in social interactions, unraveling the distinct processing mechanism of each emotion under stress conditions will deepen our understanding of the 
variability in interpretation and behavioral aspects in social interactions. Modern life mainly challenges humans with psychosocial stressors. Therefore, the present study used the most validated measure of psychosocial stress, namely the TSST, which has been proven to elicit the highest psychological and physiological responses to stress (Skoluda et al., 2015). Nevertheless, people vary in the way they perceive psychosocial stress. Future studies should examine this variable together with various types of stressor and emotion valence in order to explore this ensemble and its role in individual differences in emotion recognition under stressful social situations.

\section{References}

Bannerman, R. L., Milders, M., \& Sahraie, A. (2010). Attentional Bias to Brief Threat-Related Faces Revealed by Saccadic Eye Movements. Emotion, 10, 733-738. https://doi.org/10.1037/a0019354

Baron-Cohen, S. (2003). The Essential Difference. The Truth about the Male and Female Brain. New York: Basic Books.

Baron-Cohen, S., Wheelwright, S., Hill, J., Raste, Y., \& Plumb, I. (2001). The "Reading the Mind in the Eyes" Test Revised Version: A Study with Normal Adults, and Adults with Asperger Syndrome or High-Functioning Autism. Journal of Child Psychology and Psychiatry, 42, 241-251. https://doi.org/10.1111/1469-7610.00715

Bechtoldt, M. N., \& Schneider, V. K. (2016). Predicting Stress from the Ability to Eavesdrop on Feelings: Emotional Intelligence and Testosterone Jointly Predict Cortisol Reactivity. Emotion, 16, 815-825. https://doi.org/10.1037/emo0000134

Blair, R. J. R. (1995). A Cognitive Developmental Approach to Morality: Investigating the Psychopath. Cognition, 57, 1-29. https://doi.org/10.1016/0010-0277(95)00676-P

Brown, W. M., \& Moore, C. (2002). Smile Asymmetries and Reputation as Reliable Indicators of Likelihood to Cooperate: An Evolutionary Analysis. In S. P. Shohov (Ed.), Advances in Psychology Research (Vol. 11, pp. 59-78). Huntington, NY: Nova Science Publishers.

Corbett, B., Weinberg, L., \& Duarte, A. (2017). The Effect of Mild Acute Stress during Memory Consolidation on Emotional Recognition Memory. Neurobiology of Learning and Memory, 145, 34-44. https://doi.org/10.1016/j.nlm.2017.08.005

Curtis, W. J., \& Cicchetti, D. (2013). Affective Facial Expression Processing in 15-Month-Old Infants Who Have Experienced Maltreatment: An Event-Related Potential Study. Child Maltreatment, 18, 140-154.

https://doi.org/10.1177/1077559513487944

Cusi, A. M., Nazarov, A., Holshausen, K., Macqueen, G. M., \& McKinnon, M. C. (2012). Systematic Review of the Neural Basis of Social Cognition in Patients with Mood Disorders. Journal of Psychiatry and Neuroscience, 37, 154-169.

https://doi.org/10.1503/jpn.100179

Deckers, J. W., Lobbestael, J., van Wingen, G. A., Kessels, R. P., Arntz, A., \& Egger J. I. (2015). The Influence of Stress on Social Cognition in Patients with Borderline Personality disorder. Psychoneuroendocrinology, 52, 119-129.

https://doi.org/10.1016/j.psyneuen.2014.11.003

Hamann, S. (2001). Cognitive and Neural Mechanisms of Emotional Memory. Trends in Cognitive Sciences, 5, 394-400. https://doi.org/10.1016/S1364-6613(00)01707-1

Hänggi, Y. (2004). Stress and Emotion Recognition: An Internet Experiment Using Stres- 
sinduction. Swiss Journal of Psychology, 63, 113-125.

https://doi.org/10.1024/1421-0185.63.2.113

Herridge, M. L., Harrison, D. W., Mollet, G. A., \& Shenal, B. V. (2004). Hostility and Facial Affect Recognition: Effects of a Cold Pressor Stressor on Accuracy and Cardiovascular Reactivity. Brain \& Cognition, 55, 564-571.

https://doi.org/10.1016/j.bandc.2004.04.004

Huang, S.-L., Chang, Y.-C., \& Chen, Y.-J. (2011). Task-Irrelevant Angry Faces Capture Attention in Visual Search While Modulated by Resources. Emotion, 11, 544-552. https://doi.org/10.1037/a0022763

Kirkland, R. A., Peterson, E., Baker, C. A., Miller, S., \& Pulos, S. (2013). Meta-Analysis Reveals Adult Female Superiority in Reading the Mind in the Eyes Test. North American Journal of Psychology, 15, 121-146.

Kirschbaum, C., Pirke, K.-M., \& Hellhammer, D. H. (1993). The “Trier Social Stress Test” -A Tool for Investigating Psychobiological Stress Responses in a Laboratory Setting. Neuropsychobiology, 28, 76-81. https://doi.org/10.1159/000119004

LaBar, K. S., \& Cabeza, R. (2006). Cognitive Neuroscience of Emotional Memory. Nature Reviews, Neuroscience, 7, 54-64. https://doi.org/10.1038/nrn1825

Li, S., Weerda, R., Milde, C., Wolf, O. T., \& Theil, C. M. (2014). Effects of Acute Psychosocial Stress on Neural Activity to Emotional and Neutral Faces in a Face Recognition Memory Paradigm. Brain Imaging and Behavior, 8, 598-610. https://doi.org/10.1007/s11682-013-9287-3

Lieslehto, J., Kiviniemi, V., Mäki, P., Koivukangas, J., Nordström, T., Miettunen, J., \& Paus, T. (2017). Early Adversity and Brain Response to Faces in Young Adulthood. Human Brain Mapping, 38, 4470-4478. https://doi.org/10.1002/hbm.23674

Lupien, S. J., Maheu, F., Tu, M., Fiocco, A., \& Schramek, T. E. (2007). The Effects of Stress and Stress Hormones on Human Cognition: Implications for the Field of Brain and Cognition. Brain \& Cognition, 65, 209-237.

https://doi.org/10.1016/j.bandc.2007.02.007

Markovits, H., Trémolière, B., \& Blanchette, I. (2018). Reasoning Strategies Modulate Gender Differences in Emotion Processing. Cognition, 170, 76-82.

https://doi.org/10.1016/j.cognition.2017.09.012

Marsh, A. A., Ambady, N., \& Kleck, R. E. (2005). The Effects of Fear and Anger Facial Expressions on Approach- and Avoidance-Related Behaviors. Emotion, 5, 119-124. https://doi.org/10.1037/1528-3542.5.1.119

McEwen, B. S. (2007). Physiology and Neurobiology of Stress and Adaptation: Central Role of the Brain. Physiological Reviews, 87, 873-904. https://doi.org/10.1152/physrev.00041.2006

Oei, N. Y., Veer, I. M., Wolf, O. T., Spinhoven, P., Rombouts, S. A., \& Elzinga, B. M. (2012). Stress Shifts Brain Activation towards Ventral "affective" Areas during Emotional Distraction. Social Cognitive and Affective Neuroscience, 7, 403-412. https://doi.org/10.1093/scan/nsr024

Preuschoft, S. (1999). Are Primates Behaviorists? Formal Dominance, Cognition, and Free-Floating Rationales. Journal of Comparative Psychology, 113, 91-95.

https://doi.org/10.1037/0735-7036.113.1.91

Reed, L. I., \& DeScioli, P. (2017). Watch Out! How Fearful Face Adds Credibility to Warning of Danger. Evolution and Human Behavior, 38, 490-495. https://doi.org/10.1016/j.evolhumbehav.2017.03.003

Roozendaal, B., Okuda, S., de Quervain, D.J., \& McGaugh, J.L. (2006) Glucocorticoids 
Interact with Emotion-Induced Noradrenergic Activation in Influencing Different Memory Functions. Neuroscience, 138, 901-910.

https://doi.org/10.1016/j.neuroscience.2005.07.049

Sacco, D. F., \& Hugenberg, K. (2009). The Look of Fear and Anger: Facial Maturity Modulates Recognition of Fearful and Angry Expressions. Emotion, 9, 39-49. https://doi.org/10.1037/a0014081

Schoof, D., Preuß, D., \& Wolf, O. T. (2008). Psychosocial Stress Induces Working Memory Impairments in a n-Back Paradigm. Psychoneuroendocrinology, 33, 643-653. https://doi.org/10.1016/j.psyneuen.2008.02.004

Shields, G. S., Sazma, M. A., \& Yonelinas, A. P. (2016). The Effects of Acute Stress on Core Executive Functions: A Meta-Analysis and Comparison with Cortisol. Neuroscience and Biobehavioral Reviews, 68, 651-668.

https://doi.org/10.1016/j.neubiorev.2016.06.038

Skoluda, N., Strahler, J., Schlotz, W., Niederberger, L., Marques, S., Fischer, S., \& Nater, U. M. (2015). Intra-Individual Psychological and Physiological Responses to Acute Laboratory Stressors of Different Intensity. Psychoneuroendocrinology, 51, 227-236. https://doi.org/10.1016/j.psyneuen.2014.10.002

Smeets, T., Dziobek, I., \& Wolf, O. T. (2009). Social Cognition under Stress: Differential Effects of Stress-Induced Cortisol Elevations in Healthy Young Men and Women. Hormones \& Behavior, 55, 507-513. https://doi.org/10.1016/j.yhbeh.2009.01.011

Staal, M. A. (2004). Stress, Cognition, and Human Performance: A Literature Review and Conceptual Framework. Moffett Field, CA: NASA Ames Research Center.

Tottenham, N., Tanaka, J. W., Leon, A. C., McCarry, T., Nurse, M., Hare, T.A., \& Nelson, C. (2009). The NimStim Set of Facial Expressions: Judgments from Untrained Research Participants. Psychiatry Research, 168, 242-249. https://doi.org/10.1016/j.psychres.2008.05.006

van Marle, H. J. F., Hermans, E. J., Qin, S., \& Fernández, G. (2009). From Specificity to Sensitivity: How Acute Stress Affects Amygdala Processing of Biologically Salient Stimuli. Biological Psychiatry, 66, 649-655. https://doi.org/10.1016/j.biopsych.2009.05.014

Wolf, O. T. (2006). Effects of Stress Hormones on the Structure and Function of the Human Brain. Expert Review of Endocrinology \& Metabolism, 1, 623-632. https://doi.org/10.1586/17446651.1.5.623

Wolf, O. T., Schulte, J. M., Drimalla, H., Hamacher-Dang, T. C., Knoch, D., \& Dziobek, I. (2015). Enhanced Emotional Empathy after Psychosocial Stress in Young Healthy Men. Stress, 18, 631-637. https://doi.org/10.3109/10253890.2015.1078787 